\title{
Impact of Weekend Physiotherapy Service on the Cost Effectiveness of Elective Orthopaedic Hip and Knee Arthroplasty
}

\author{
I.P. Pengas ${ }^{*}, 1$, W.S. Khan ${ }^{2}$, C.A. Bennett ${ }^{3}$ and K.S. Rankin ${ }^{1}$ \\ ${ }^{1}$ Department of Orthopaedics, Perth Royal Infirmary, Perth, Scotland PH1 1NX, UK \\ ${ }^{2}$ University College London Institute of Orthopaedics \& Musculoskeletal Sciences, Royal National Orthopaedic \\ Hospital, Stanmore, London HA7 4LP, UK \\ ${ }^{3}$ Department of Physiotherapy, Perth Royal Infirmary, Perth, Scotland PHI INX, UK
}

\begin{abstract}
We performed a prospective correlational study to evaluate the efficiency and cost effectiveness of weekend physiotherapy in accelerating rehabilitation, reducing hospital stay as well as hospital costs for joint arthroplasty patients in a busy Scottish district general hospital. Patients that underwent elective hip (470) and knee (321) arthroplasty were analysed over a 12 month period. A four month period with weekend physiotherapy provision was arranged to ascertain its effectiveness on the length of stay and the achievement of set physiotherapy milestones. Data collected included length of stay and progression in a defined set of physiotherapy milestones. The relationship between time to discharge, mobilisation with sticks, straight leg raise, $90^{\circ}$ knee flexion and cost effectiveness of service were used to determine the correlation, and analysis of the interactions of these factors separately. Our Outcome data demonstrate a statistical significance for the time to mobilisation with two sticks for hip $(\mathrm{p}=0.0030)$ and knee $(\mathrm{p}=0.0037)$ arthroplasty patients. There was a trend towards earlier discharge times for all patients receiving weekend physiotherapy, but this was not statistically significant. We conclude that the provision of a continuous programme of weekend physiotherapy for all arthroplasty patients has the potential benefit of a quicker rehabilitation that would results in a cost saving.
\end{abstract}

Keywords: Arthroplasty, cost-effective, orthopaedics, physiotherapy.

\section{INTRODUCTION}

Osteoarthritis is a common cause of pain and disability particularly in the elderly [1]. Hip and knee arthroplasty that have become common interventions for severe joint disease, and their utilisation is increasing in the United Kingdom [2]. These joint replacement procedures provide effective pain relief and increased function for patients, thus improving their quality of life [3]. In the United Kingdom (UK), there are over 80,000 total hip and 80,000 knee arthroplasty procedures carried out annually [4]. Furthermore, these figures are expected to increase sharply over the next two or three decades due to our ageing population and the lowering of the threshold for offering arthroplsty in younger patients. A significant proportion of the costs per patient are due to the length of hospital stay with an estimated annual cost of $£ 70$ million for total knee arthroplasty alone [5]. In fact, the patients' time in hospital accounts for approximately $50 \%$ of the total cost of a standard joint replacement, with the average cost of a bed per night averaging $£ 687$ [6].

There have been very few studies addressing the impact weekend physiotherapy and discharge, yet there is a potential for large savings on the average cost of hip and knee Arthroplasty [7]. Many orthopaedic units in the United Kingdom do not have access to continuous physiotherapy services during the weekend for patients undergoing hip or

*Address correspondence to this author at the Department of Orthopaedics, Perth Royal Infirmary, Perth, Scotland; Tel: +44 7894507181;

E-mail: yiannispengas@yahoo.com knee arthroplasty, and this may contribute for times to discharge. According to the British Orthopaedic Association, patients undergoing knee replacement at the end of the working week should have access to physiotherapy at the weekend [8]. For example, patients who receive arthroplasty earlier in the week benefit from it for at least two days of physiotherapy post-operatively, while patients undergoing their procedures in the latter part of the week have at the most one day of physiotherapy prior to the weekend. On observation in our unit, patients who receive arthroplasty earlier in the week appear to mobilise and discharge earlier than those who had procedures performed later in the week.

The aim of this study was to demonstrate that patients receiving physiotherapy over the weekend show quicker rehabilitation and therefore have speed recovery than those patients whom receive no physiotherapy over this period. The potential benefits include a decrease in hospital costs which would generate more throughput, reduce waiting lists, have less impact on nursing staff work load and maximise post-operative results with increased patient satisfaction.

\section{METHODS}

Data was prospectively collected over a 12 month period for all hip and knee arthroplasty patients including those who went through undergoing hip resurfacing and unicompartmental knee procedures. To establish the effectiveness of weekend physiotherapy, a pilot study of four months covering 15 weekends was commenced. Physiotherapy for a three hour session per day was provided by a technical 
instructor (physiotherapy assistant) in that time period. Data included demographic details, the day of the week the operation took place, the type of operation and their time to achieve their physiotherapy milestones. Physiotherapy milestones utilised in the study included progression to straight leg raise (SLR), at $90^{\circ}$ knee flexion, mobilisation with sticks and length of stay for the knee arthroplasties. In addition, hip arthroplasties were monitored by progression to sticks and length of stay. The range of movement (ROM) was also recorded. Thus, comparison of the two groups that opted for the same surgeon/firm and operative day in the week by their physiotherapy milestones achievements and their length of hospital stay were analysed.

The exclusion criteria included complex, bilateral or revision arthroplasty, patient stay over 10 days due to infection or social circumstances and complications preventing mobilisation e.g. pulmonary embolus.

Statistical Package for the Social Sciences (SPSS) Version 15 (SPSS Inc., 233 S. Wacker Drive, 11th floor Chicago, Illinois 60606) was utilized to implement the analysis of the data in this study using the Mann-Whitney U test.

\section{RESULTS}

The mean age for all hip patients was 65.21 years (SD 11.64 years, range $27-89$ years) and 70.95 years (SD 8.91 years, range 49-92 years) for the knee patients. There were 235 females and 235 males in the hip group, and 147 females and 174 males in the knee group.

\section{Hip Arthroplasty}

Data for hip arthroplasty patients on days to mobilise with two sticks and days to discharge are shown in Table $\mathbf{1 .}$ Analysis of this data showed the mean times for mobilising on two sticks (3.53 days versus 3.11 days) and discharge (5.22 days versus 4.95 days) were lower for those patients who received weekend physiotherapy. This was statistically significant $(\mathrm{p}=0.0030)$ for mobilising on two sticks but not for discharge $(\mathrm{p}=0.2071)$ (Fig. 1).

\section{Knee Arthroplasty}

Data for knee arthroplasty patients on days to mobilise with two sticks and days to discharge are shown in Table 2. Analysis of these data revealed that patients receiving weekend physiotherapy mobilised significantly earlier with a mean time to mobilising on sticks of 3.29 days compared to the group that did not receive physiotherapy whose mean time was 3.87 days $(p=0.0037)$. Those patients who received weekend physiotherapy also had a lower mean time to discharge (5.45 days versus 5.04 days) which was not statistically significant ( $\mathrm{p}=0.0534)$ (Fig. 2).

Table 1. Days to mobilise and discharge in the hip patient group.

\begin{tabular}{|c|c|c|c|c|}
\hline & $\mathbf{N}$ & Mean & SEM & $P$ Value \\
\hline Weekend physiotherapy: Days to mobilise with two sticks & 113 & 3.106 & 0.130 & 0.0030 \\
\hline No weekend physiotherapy: Days to mobilise with 2 sticks & 357 & 3.529 & 0.077 & \\
\hline Weekend physiotherapy: Days to discharge & 114 & 4.947 & 0.142 & 0.2071 \\
\hline No weekend physiotherapy: Days to discharge & 353 & 5.224 & 0.091 & \\
\hline
\end{tabular}

(a)

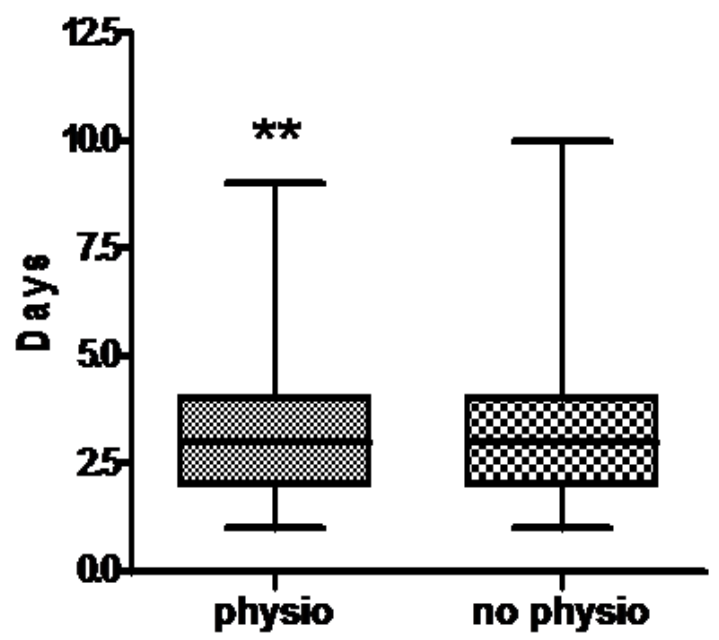

(b)

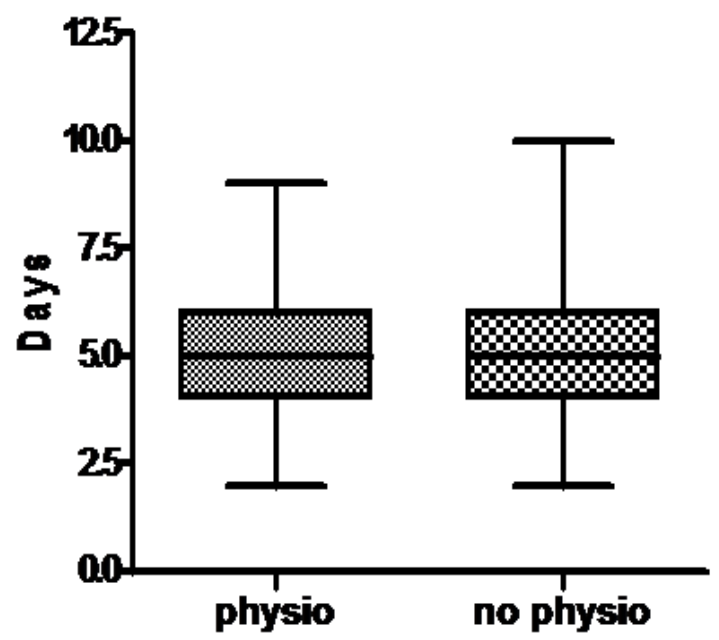

Fig. (1) Following hip arthroplasty patients receiving weekend physiotherapy mobilised significantly earlier, (a) $(* * \mathrm{p}=0.0030)$, but did not discharge significantly earlier (b). 
Table 2. Days to mobilise and discharge in the knee patient group.

\begin{tabular}{|c|c|c|c|c|}
\hline & $\mathbf{N}$ & Mean & SEM & $P$ Value \\
\hline Weekend physiotherapy: Days to mobilise with two sticks & 77 & 3.286 & 0.127 & 0.0037 \\
\hline No weekend physiotherapy: Days to mobilise with two sticks & 240 & 3.867 & 0.096 & \\
\hline Weekend physiotherapy: Days to discharge & 78 & 5.038 & 0.191 & 0.0534 \\
\hline No weekend physiotherapy: Days to discharge & 243 & 5.453 & 0.105 & \\
\hline
\end{tabular}

(a)

\section{Kneestosids}

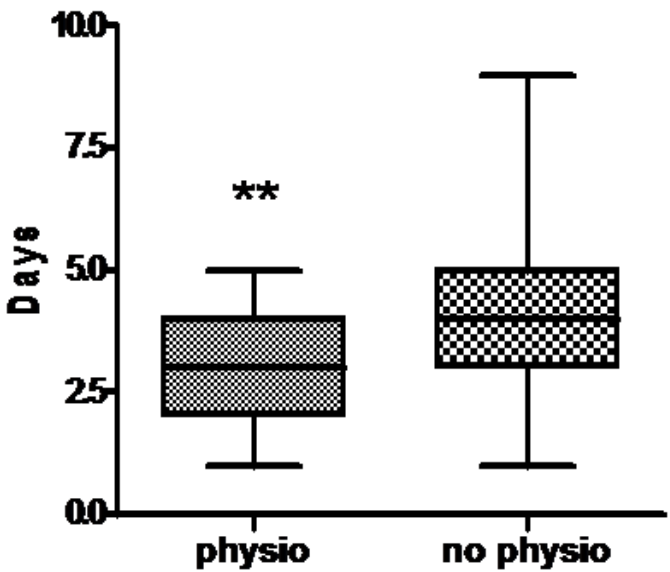

(b)

\section{Knees bodschigge}

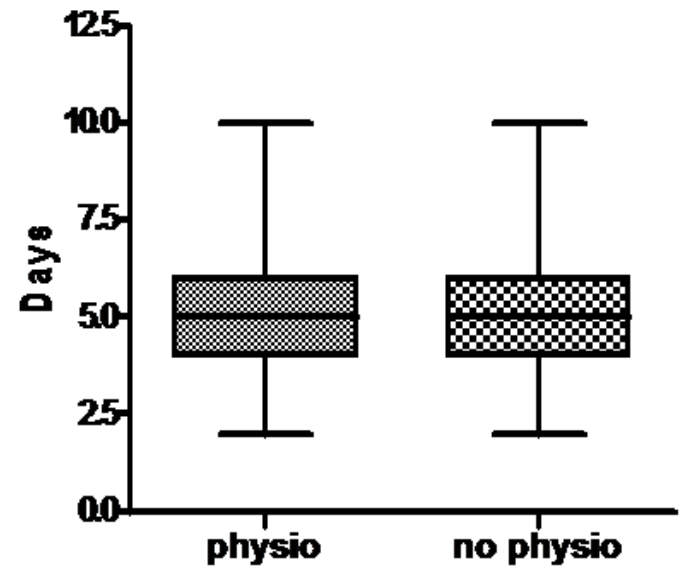

Fig. (2) Following knee arthroplasty patients receiving weekend physiotherapy mobilised significantly earlier, (a) $(* * \mathrm{p}=0.0037)$, but did not discharge significantly earlier (b).

Interestingly, the time to achieve $90^{\circ}$ flexion (2.99 days versus 2.49 days, $\mathrm{p}=0.69)$ and SLR (2.75 days versus 2.49 days, $\mathrm{p}=0.19)$, and $\mathrm{ROM}$ on discharge $\left(86.19^{\circ}\right.$ versus $87.72^{\circ}$, $\mathrm{p}=0.22$ ) showed a trend of improvement in the group receiving weekend physiotherapy, but this was not statistically significant (Figs. 3, 4).

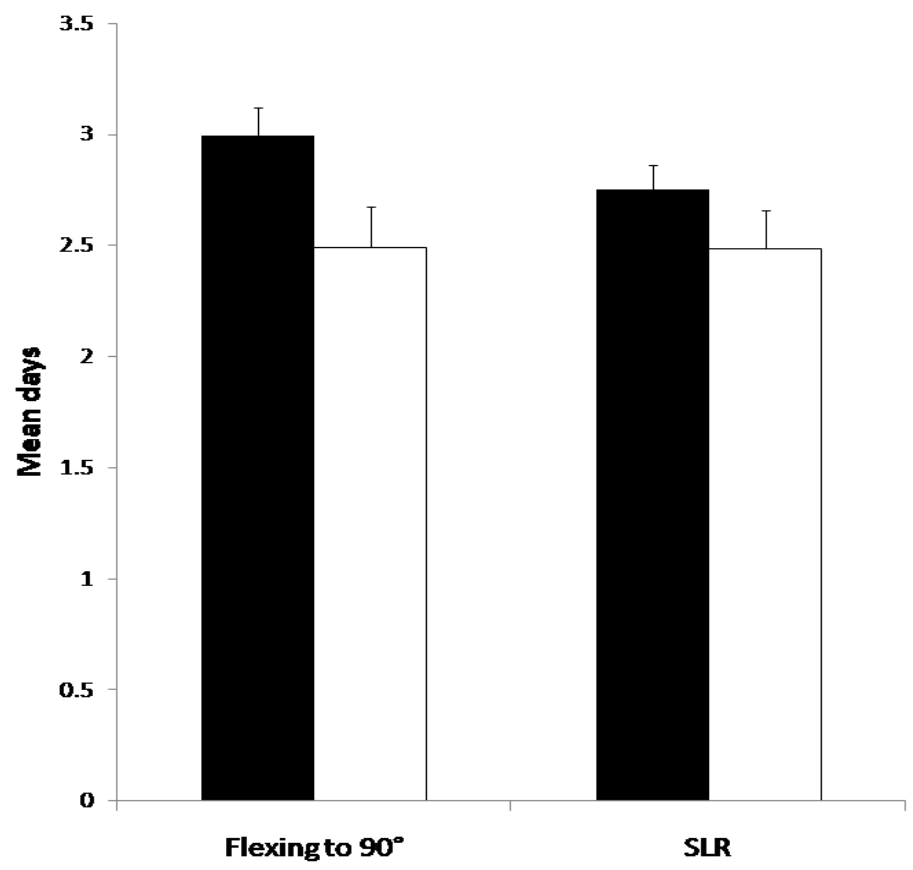

- No weekend physiotherapy

$\square$ Weekend physiotherapy

Flexing to $90^{\circ}$

SLR

Fig. (3). Days to achieve $90^{\circ}$ flexion and straight leg raise (SLR) improved with weekend physiotherapy, however this differencewas not significant. 


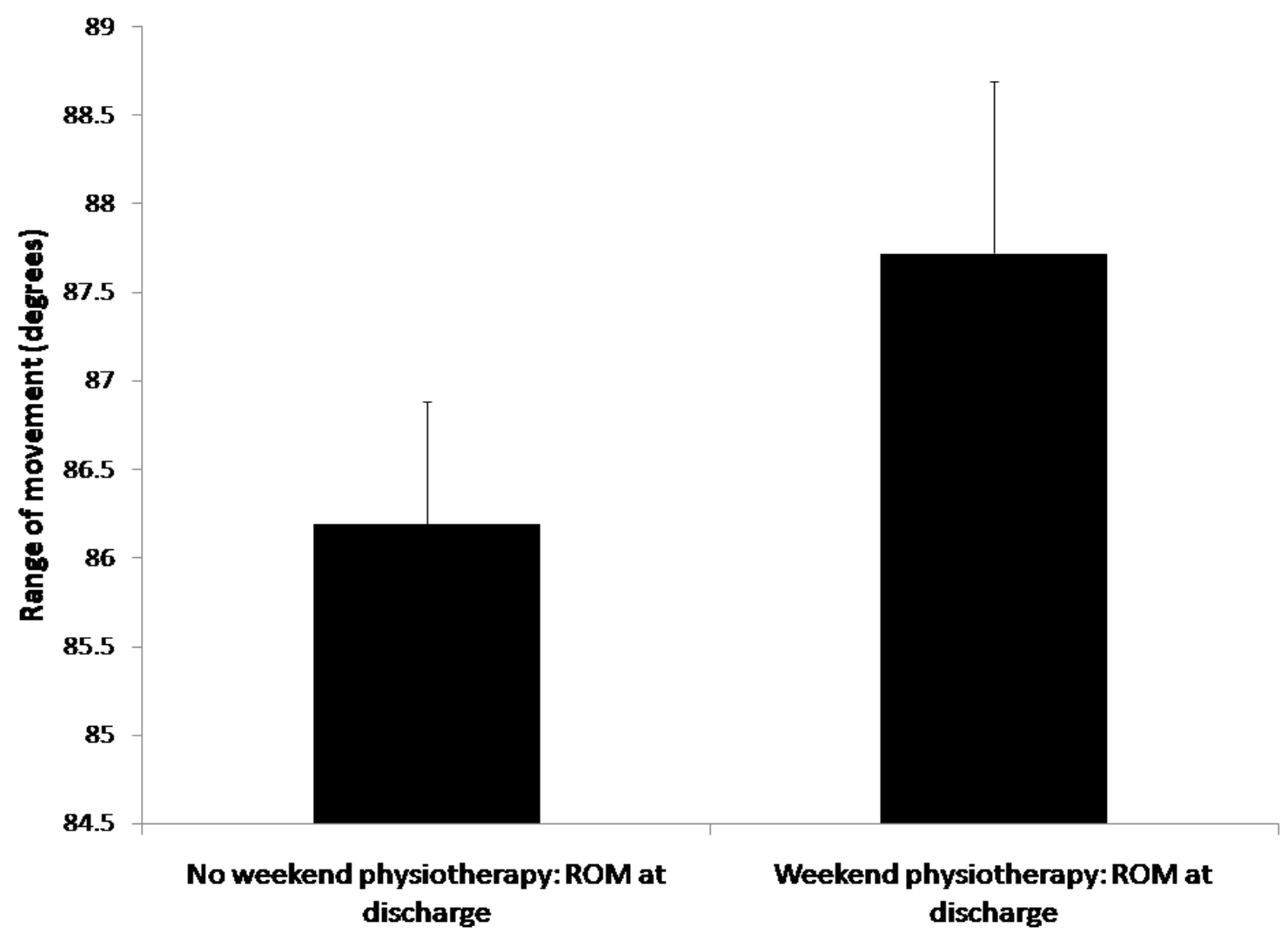

Fig. (4). Range of movement (ROM) on discharge improved with weekend physiotherapy, however this difference was not statistically significant.

\section{DISCUSSION}

The main finding of this study is that patients following hip and knee arthroplasty are significantly quicker to mobilise with sticks if they receive weekend physiotherapy compared to patients who did not receive weekend physiotherapy. There was a trend suggesting a reduced hospital stay for patients following hip and knee arthroplasty but this was not statistically significant.

The effects of weekend physiotherapy for patients receiving arthroplasty have been scarcely assessed. The most obvious reason for this is the difficulty in setting up a programme of weekend physiotherapy for a sustained period of time. The physiotherapists in our department identified the potential benefits of weekend physiotherapy and were therefore motivated to attend patients over the weekends. In order, to promote this weekend activity, physiotherapists were paid as per their normal hourly rate and spent an average of six hours each weekend (three hours per day) attending patients that amounted to $£ 200$ per weekend.

Hip arthroplasty patients tend to require less input from physiotherapy as compared to knee arthroplasty patients. The reason for this includes the ability to immediately have a good range of movement and therefore only need instructions on walking and safe movements to prevent dislocation. Knee arthroplasty patients need more physiotherapy input to achieve an adequate range of movement and regain muscle power in order to facilitate a near normal gait pattern. In addition, knee arthroplasty patients must work harder on active and passive movements to achieve this and need daily physiotherapy if they are going to mobilise faster. Therefore, it is not surprising that patients who underwent a knee arthroplasty procedure and received daily physiotherapy demonstrated improved times to $90^{\circ}$ flexion, SLR and ROM. Previous studies have reported the influence of physiotherapy interventions in the short term benefit after knee arthroplasty [9]. The knee arthroplasty group had greater function in activities of daily living (ADL) such as aided walking which could increase earlier independence. However this did not translate in to a significant reduction in time to discharge. On review of the case notes wound leakage was identified as the main reason for the delay in discharge once the patients had satisfied the physiotherapy discharge requirements i.e. mobilising safely on two sticks with adequate ROM and compliance with stairs. In order for weekend physiotherapy to be costeffective, the majority of patients receiving it should have their time to discharge reduced by at least one day. For example, in our unit the cost of the weekend physiotherapy was $£ 200$ based on overtime. This would cost $£ 10,400$ for a calendar year.

An extra day in hospital costs $£ 687$. Our results did not demonstrate statistical significance in days to discharge but the average reduction of 0.28 days for hips and 0.415 days for knees would have saved hospital's $£ 77,492$ for Hips and $£ 78,200$ for the knees. If we then add these together a total saving would be of $£ 155,692$ over the course of the study versus $£ 10,400$ required to cover the weekends. Length of stay following hip and knee arthroplasty has been decreasing [10] however, as demonstrated by this study, this can be improved upon by the simple contribution of weekend physiotherapy. 
If time from admission to discharge was improved upon, this would allow more resources to be available for other patients and disciplines that would increase throughput. It is our view that continuing physiotherapy services over the weekend improved the overall continuity of care in our arthroplasty patients enabling a faster recovery and earlier discharge. There is a significant rise in hip and knee arthroplasty predicted over the next 30 years [11]. Without extra input from allied healthcare services, orthopaedic care will become overburdened in the long-term. Thus, as proposed, additional physiotherapy staffing at the weekends has the potential to reduce time to discharge which in turn may lead to reduced waiting list times or alternatively free more beds for both trauma and elective admissions.

We recommend continuous daily physiotherapy for all arthroplasty patients regardless of day of operation. While many patients do not require a seven day physiotherapy service, the need to provide weekend physiotherapy for some patients has increased and will increase further over next few decades. The patients most at risk of delayed discharge include the group of patients operated on in the latter part of the working week and weekend physiotherapy cover at present does not adequately provide an optimum service. As hospitals strive to prioritise their resources, savings can be made through the provision of weekend physiotherapy for arthroplasty patients by reducing the bed days and nursing interventions required.

\section{CONCLUSION}

This study highlights the cost-effectiveness of weekend physiotherapy services and shows how this can help reduce the waiting lists by increasing throughput. With the expected rise in hip and knee surgeries expected to rise over the next 20 years, weekend physiotherapy will play a major role in satisfying the rehabilitation needs of the United Kingdom population and should be embraced throughout the NHS.

\section{CONFLICT OF INTEREST}

The authors confirm that this article content has no conflict of interest.

\section{ACKNOWLEDGEMENTS}

Declared none.

\section{REFERENCES}

[1] Ling SM, Fried LP, Garrett ES, Fan MY, Rantanen T, Bathon JM. Knee osteoarthritis compromises early mobility function: The Women's Health and Aging Study II. J Rheumatol 2003; 30(1): 114-20.

[2] Dixon T, Shaw M, Ebrahim S, Dieppe P. Trends in hip and knee joint replacement: socioeconomic inequalities and projections of need. Ann Rheum Dis 2004; 63(7): 825-30.

[3] Murray D. Surgery and joint replacement for joint disease. Acta Orthop Scand Suppl 1998; 281: 17-20.

[4] Ebinesan AD, Sarai BS, Walley G, Bridgman S, Maffulli N. Total knee arthroplasty: good agreement of clinical severity scores between patients and consultants. BMC Musculoskelet Disord 2006; 7: 61 .

[5] From National Joint Registry. www.njrcentre.org.uk

[6] Birrell F, Johnell O, Silman A. Projecting the need for hip replacement over the next three decades: influence of changing demography and threshold for surgery. Ann Rheum Dis 1999; 58(9): 569-72.

[7] Hughes K, Kuffner L, Dean B. Effect of weekend physical therapy treatment on postoperative length of stay following total hip and total knee arthroplasty. Physiother Can 1993; 45(4): 245-9.

[8] Total Hip Replacement: A Guide to Best Practice, British Orthopaedic Association. http://www.boa.ac.uk/BOAAhome.htm [Accessed 18th November 2003].

[9] Minns Lowe CJ, Barker KL, Dewey M, Sackley CM. Effectiveness of physiotherapy exercise after knee arthroplasty for osteoarthritis: systematic review and meta-analysis of randomised controlled trials. BMJ 2007; 335(7624): 812.

[10] Dowsey MM, Kilgour ML, Santamaria NM, Choong PF. Clinical pathways in hip and knee arthroplasty: a prospective randomised controlled study. Med J Aust 1999; 170(2): 59-62.

[11] Singh JA. Epidemiology of knee and hip arthroplasty: A systematic review. Open Orthop J 2011; 5: 80-85. 